\title{
OPTIMUM PATH DETERMINATION TO FACILITATE FIRE STATION RESCUE MISSIONS USING ANT COLONY OPTIMIZATION ALGORITHMS (CASE STUDY: CITY OF KARAJ)
}

\author{
N. Zarrinpanjeh ${ }^{1}$, F. Dadrass Javan ${ }^{2,4 *}$, A. Naji ${ }^{3}$, H. Azadi ${ }^{4}$, P. De Maeyer ${ }^{4}$, F. Witlox ${ }^{4}$ \\ ${ }^{1}$ Department of Geomatics Engineering, Qazvin Branch, Islamic Azad University, Qazvin, Iran - nzarrin@qiau.ac.ir \\ ${ }^{2}$ Department of Surveying and Geomatics Engineering, University College of Engineering, University of Tehran, Tehran, Iran - \\ fdadrasjavan@ut.ac.ir \\ 3 Department of Geomatics Engineering, Ramsar Branch, Islamic Azad University, Ramsar, Iran - Amirmasoud.naji@gmail.com \\ ${ }^{4}$ Department of Geography, Ghent University, Ghent, Belgium \{farzaneh.dadrassjavan, hossein.azadi, frank.witlox, \\ philippe.demaeyer\}@ugent.be
}

Commission III, ICWG III/IVa

KEY WORDS: Ant Colony Optimization, Optimum Path Generation, Fire Station, Urban Road Network

\begin{abstract}
:
The successful conduct of a rescue mission in urban areas is directly related to the timely deployment of equipment and personnel to the incident location which justifies the quest for optimum path selection for emergency purposes. In this study, it is attempted to use Ant Colony Optimization (ACO) to find the optimum paths between fire stations and incident locations. It is also attempted to build up an evaluation tool using ACO to detect critical road segments that the overall accessibility to fire station services throughout the urban area is constituted upon their excellent functionality. Therefore, an ACO solution is designed to find optimum paths between the fire station and some randomly distributed incident locations. Regarding different variants of ACO, the algorithm enjoys the Simple Ant Colony Optimization deployment strategy combined with Ant Algorithm Transition rules. Iteration best pheromone updating is also used as the pheromone reinforcement strategy. The cost function used to optimize the path considers the shortest Euclidean distance on the network. The results explicitly state that the proposed method is successful to create the optimum path in 95.45 percent of all times, compared to Dijkstra deterministic approaches. Moreover, the pheromone map as an indicator of the criticality of road elements is generated and discussed. Visual inspection shows that the pheromone map is verified as the road criticality map concerning fire station access to the region and therefore pre-emptive measures can be defined by analyzing the generated pheromone map.
\end{abstract}

\section{INTRODUCTION}

Optimum path selection for rescue missions is believed to be the matter of life and death when every moment is vital to save human lives and to prevent the expansion of disaster, especially in urban areas. Conducting a fully successful rescue mission in terms of the least possible loss of life and damage necessitates the fast deployment of equipment and personnel to the incident location. Therefore, in case of any incident, firefighters as pioneer rescuers in urban areas must take the best possible path to the location. Therefore, before any action about accidents, the fire department should study the shortest paths to their service area and also gather information about critical road segments which are mostly used in the missions.

The Optimum Path problem has always been a hot topic in mathematics and geoinformatics. The shortest path is another term to name the problem where the main goal only considers the Euclidian distance on the network. On the contrary, Optimum path may consider costs such as elapsed time or traffic delays or even pavement conditions. Optimum path problem is considered as an optimization problem trying to generate the optimum route between start and destination points enjoying minimum sum of weights of constituent links. Therefore, attempts have been made to provide solutions enjoying efficiency and accuracy at the same time. Generally, Optimization algorithms are roughly divided into deterministic and stochastic algorithms. Among deterministic approaches, Dijkstra (Dijkstra, 1959), BellmanFord algorithm (Bellman, 1958), A* search algorithm (Zeng, Church 2009), Floyd-Warshall algorithm (Floyd, 1962) and Johnson's algorithm (Johnson, 1977) can be listed. In the stochastic category, some evolutionary approaches such as Genetic Algorithms and Ant Colony Optimization (ACO) have been used.

Genetic Algorithm, a pioneering evolutionary optimization algorithm, has been studied to fit the shortest path problem. Syarif et al. (2018) proposed a solution for the shortest path problem using genetic algorithms. In this approach cost values are represented by Triangular Fuzzy Numbers, adopting the concept of ranking fuzzy numbers to determine how good the solutions are. The results were promising on small networks (Syarif et al. 2018). Behera et al. (2018) investigated the use of Genetic algorithms for the shortest path routing problem. They found the genetic algorithm more scalable and insensate to variations in topologies (Behera et al. 2020). In recent research, a self-adaptive genetic algorithm by adjusting the encoding parameters is proposed (Liu et al. 2016). The experiments indicated that the improved genetic algorithm DRSP-GA could obtain better solutions in terms of adapting to new transportation more rapidly than $\mathrm{A}^{*}$ algorithm and Dijkstra algorithm in the shortest path problem (Liu et al. 2016).

\footnotetext{
* Corresponding author
} 
ACO solution has also been studied to fit optimum path problems although fewer attentions have been paid compared to other applications of ACO. As one of the pioneering studies, ACO is applied for routing and road network balancing (Sim and Sun 2003). In this survey, three major ACO methods were compared to each other, and the stagnation problem was also discussed. (Sim, Sun 2003). Ghoseriri et al, (2009) proposed an ACO algorithm to solve the bi-objective shortest path problem. The results indicated that the multi-objective shortest path problem is efficiently solvable by ACO. ACO is also tested for stochastic shortest path problems where all edges are subject to noise that reflects delays and uncertainty. It was observed that when the noise is perfectly correlated, the ants find the shortest paths efficiently (Horoba, Sudholt 2010). Glabowoski et al. (2012) provided a comprehensive survey on how to use ACO to solve the shortest path problem, its elements, and parameters and also how to tune parameters (Glabowski et al. 2012). ACO is previously inspected for finding the best routes in the disasteraffected urban area (Samadzadegan et al. 2011). This study considers damage values to each road segment as a parameter in the ACO transition rule, therefore the optimum path is computed considering damages to road segments.

Although being informed of the optimum path has gained popularity among users, it is a vital item when it comes to the emergency in urban areas. From emergencies related to fire stations to post-disaster issues, having accessibility to reach the affected locations fast and safe is the main challenge. In 2013 a GIS-based fire emergency response system that considered the optimum path as an important component of the system was proposed (Forkuo, Quaye-Ballard 2013). More recently, optimum paths from tourist places to railways, Hospitals, and Fire stations are inspected (Sadhu et al. 2017).

The aggregate of all studies in the field of the optimum path selection and disaster management justifies the inspection of ACO solutions for the rescue missions. In this paper reviewing ACO basics, the proposed method for optimum path generation is defined and compared to the Dijkstra solution. Then generating critical road maps from pheromone values is explained. Finally, the proposed method is tested using case study data.

\section{ANT COLONY OPTIMIZATION FOR SHORTEST PATH PROBLEM}

Ant Colony Optimization is a meta-heuristic algorithm to solve optimization problems and is invented to be used as an optimization algorithm by Marco Dorigo (Dorigo, 1992). Since then it has been developed and gained popularity among researchers to be used as an optimization solution. ACO is inspired by the collective foraging behavior of ants where the aggregate of the behavior of unintelligent individuals results in intelligent outputs.

Consider $G(V, E)$ is the network of roads and streets comprised of $\mathrm{V}$ vertices or nodes and $\mathrm{E}$ edges or links, within which we are intended to find the shortest path between start and end nodes. The ACO solution is initiated by locating some $k$ ants on the start node. Each ant is deployed to take one of the feasible edges to the start node and relocate to a new node position in step one. The ant takes another step in conformance to deployment rules until it reached the end node consequently making a path between the start and the end nodes. Each ant chooses the next edge according to the transition rule probabilistically. Simply speaking, transition rules are entitled to favor the selection of an edge enjoying higher pheromone intensity as the main parameter and additional parameters such as the length of the edge. At the first iteration, all edges usually have the same pheromone values. Performing the same procedure to each of $k$ ants, $k$ paths are generated at the end of the first iteration. In the next step, pheromone values are reinforced at the constituent edges of all generated $k$ path or the iteration best or overall best solution according to the solution configuration in the updating procedure. To prevent premature stagnation of the solution after each iteration, the pheromone value on each edge is reduced proportionately to the current pheromone value. As the pheromone values on the shortest path are reinforced more intensively after some iterations, the final solution is converged to the shortest path. Regardless of the numerous variants of ACO, they share the fundamental elements as followings:

\subsection{Deployment}

Deployment is defined as a set of rules about how ants move all across the network which includes loop removals or blacklisting of previously chosen nodes.

\subsection{Transition Rule}

Consider $N_{i}^{k}$ to be the set of feasible nodes to node $i$ for ant $k$. In its simplest form, the probability of choosing edge $i j$ is computed according to equation (1) where $\tau_{i j}$ is the pheromone concentration at edge $i j, \mathrm{t}$ is the iteration number and, $\alpha$ is the power.

$P_{i j}^{k}(t)=\left\{\begin{array}{cc}\frac{\tau_{i j}^{\alpha}(t)}{\sum_{u \in N_{i}^{k}} \tau_{i u}^{a}(t)} & \text { if } j \in N_{i}^{k} \\ 0 & \text { if } j \in N_{i}^{k}\end{array}\right.$

The transition sue in equation (1) is used in Simple Ant Colony Optimization (Engelbrecht, 2007). Other configurations have been introduced as transition rules as stated in equation (2) and (3). In these transition rules, $\eta_{i j}$ represent some prior knowledge about the edge such as its length.

$$
\begin{aligned}
& P_{i j}^{k}(t)=\left\{\begin{array}{cc}
\frac{\tau_{i j}^{\alpha}(t) \eta_{i j}^{\beta}(t)}{\sum_{u \in N_{i}^{k}} \tau_{i u}^{\alpha}(t) \eta_{i u}^{\beta}(t)} & \text { if } j \in N_{i}^{k} \\
0 & \text { if } j \notin N_{i}^{k}
\end{array}\right. \\
& P_{i j}^{k}(t)=\left\{\begin{array}{cc}
\frac{\alpha \tau_{i j}(t)(1-\alpha) \eta_{i j}(t)}{\sum_{u \in N_{i}^{k}\left(\alpha \tau_{i u}(t)(1-\alpha) \eta_{i u}(t)\right)}} & \text { if } j \in N_{i}^{k} \\
0 & \text { if } j \notin N_{i}^{k}
\end{array}\right.
\end{aligned}
$$

\subsection{Updating}

At this stage, the pheromone values on each edge are reinforced according to the desirability of the constructed path. The rule of thumb indicated that pheromone values reinforcement should be proportionate to the inverse of the length of the constructed path (Engelbrecht, 2007). A different variant of ACO proposes different updating solution. Some variants consider all the constructed path for updating while the others apply only the iteration best or the overall best. Besides choosing the updating path, the amount of pheromone updating is also a matter of debate. Quality, Quantity, and Density-based solutions have been used in different variants of ACO (Engelbrecht, 2007). 


\subsection{Evaporation}

Evaporation is defined as the reduction of pheromone values after each iteration. Consider $\rho$ as a positive value smaller than 1 , the pheromone values for the next iteration are usually multiplied to $(1-\rho)$ as mention in equation (4).

$\tau_{i j}(t+1) \leftarrow(1-\rho) \tau_{i j}(t)$

\subsection{Convergence}

Convergence has always been a controversial concept in all the iterative meta-heuristic solution. In ACO, convergence is usually acquired when all or a majority of $k$ ants at each iteration construct the same path.

\section{THE PROPOSED METHOD}

In this paper, an ACO solution for finding the shortest paths for fire station services is proposed. At first road network and fire station locations along with random incident locations, are introduced to preprocessing where data is prepared for ACO solution. Then, the ACO based solution is performed and the shortest path from the fire station to each incident location is generated. The results are then compared to the path created by the deterministic Dijkstra algorithm. Moreover, the pheromone values of all solutions are aggregated into the pheromone concentration maps which leads to the mapping of the critical roads for fire-station services.

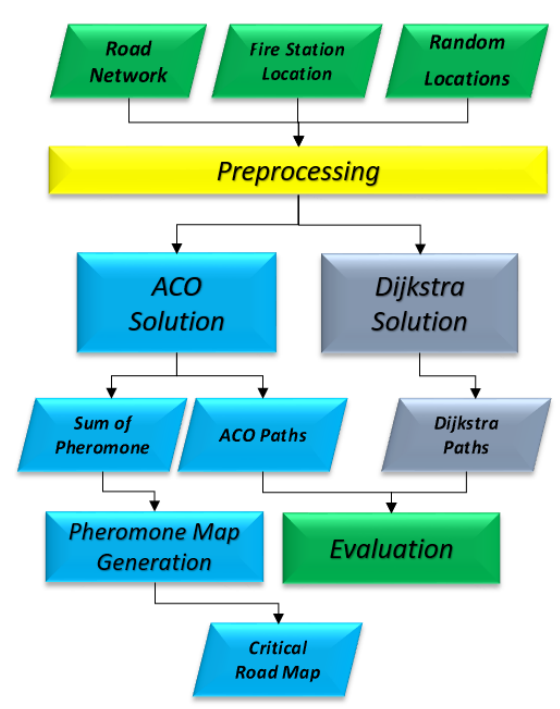

Figure 1. The flowchart of the proposed method.

\subsection{Input Data and Pre-processing}

Input Data comprised of the network of city roads and Firestation locations along with incident locations. Regarding preprocessing of the road network, the following items should be mentioned:

i. Resolving GIS-ready related errors such as overshoot and undershoot and loose ends.

ii. Map generalization, which includes the process of removing the unnecessary nodes and consequently the redundant edges.
Regarding fire station locations and incident locations the main pre-processing concerns the verification of their location on the network.

\subsection{ACO Solution}

The proposed ACO solution considers a random deployment of ants as practiced in SACO (Dorigo, Di Caro 1999). Therefore, ants are placed at the fire station locations and they are randomly deployed to reach the target incident location. Concerning the transition rule, the algorithm is designed to uses the transition rule mentioned in equation (3)(Dorigo, 1992). The pheromone updating is built upon the best iteration solution and the AntCycle solution which considers a $\mathrm{Q}$ value to tune the reinforcement rate according to equation (5) (Engelbrecht, 2007). In this equation, $f\left(x^{k}(t)\right)$ equals the inverse of the length of the path. The evaporation procedure is designed to multiply the amount of pheromone to (1- $\rho$ ) after each iteration. Finally, the algorithm is converged when all deploying ants construct the same path.

$\Delta \tau_{\mathrm{ij}}^{k}(\mathrm{t})\left\{\begin{array}{cc}Q f\left(x^{k}(t)\right) & \text { if link }(i j) \text { occures in } x^{k}(t) \\ 0 & \text { Otherwise }\end{array}\right.$

\subsection{Dijkstra Solution}

The deterministic Dijkstra solution used to evaluate the ACO algorithm is the exact recreation of the classic Dijkstra Solution (Cormen et al. 2009). The solution is chosen to produce reference shortest paths for the intention of evaluation.

To conduct our experiment, ACO is individually applied to find the shortest path between the fire station and incident location and the shortest path, and the final pheromone value at the last iteration is also recorded. The generated paths are compared to the results of Dijkstra solutions.

\subsection{Pheromone Map}

The sum of pheromone values for each run of the algorithm is computed and mapped into the critical road map. The generated map defines road which has been selected by ants several times not only on the constructed path but also during the whole process of ACO. The maps show which road segments are the most critical transportation lifelines for the benefit of the fire department. In other words, the intensification of the sum of pheromone value on a road segment is interpreted as the segment has been chosen more frequently in the constructed best path (according to iteration best Pheromone Updating paradigm). Aggregating pheromone values of all paths, the overall utilization of segment by ants is generated and converted into a map as depicted in figure 1 . The generated map demonstrates how critical each road segment is to run rescue missions from the selected fire station throughout the region. All road segments are accordingly categorized concerning the criticality of their role in providing access from the fire station to the incident location.

\section{EXPERIMENTS AND RESULTS}

To evaluate the proposed strategy, a toolbox is designed and developed to encompass all prominent ACO variants enjoying the capability of blending different ACO elements and configurations. This toolbox which is capable of providing 
solutions for different problems is exclusively adjusted for the shortest path problem.

\subsection{The Case Study Area and Pre-processing}

To test the capability of the proposed method, experiments are conducted to the fire station and road network of Azimieh District in Karaj, Alborz Province, Iran. The region is a residential area and located in North West of the city of Karaj.

Preprocessing according to the road network and fire station and also incident locations are applied. The network of the roads Fire station and incident locations are depicted in figure 2. It also includes the removal of unnecessary nodes due to the map scale which facilitated ant deployment in the next stage.

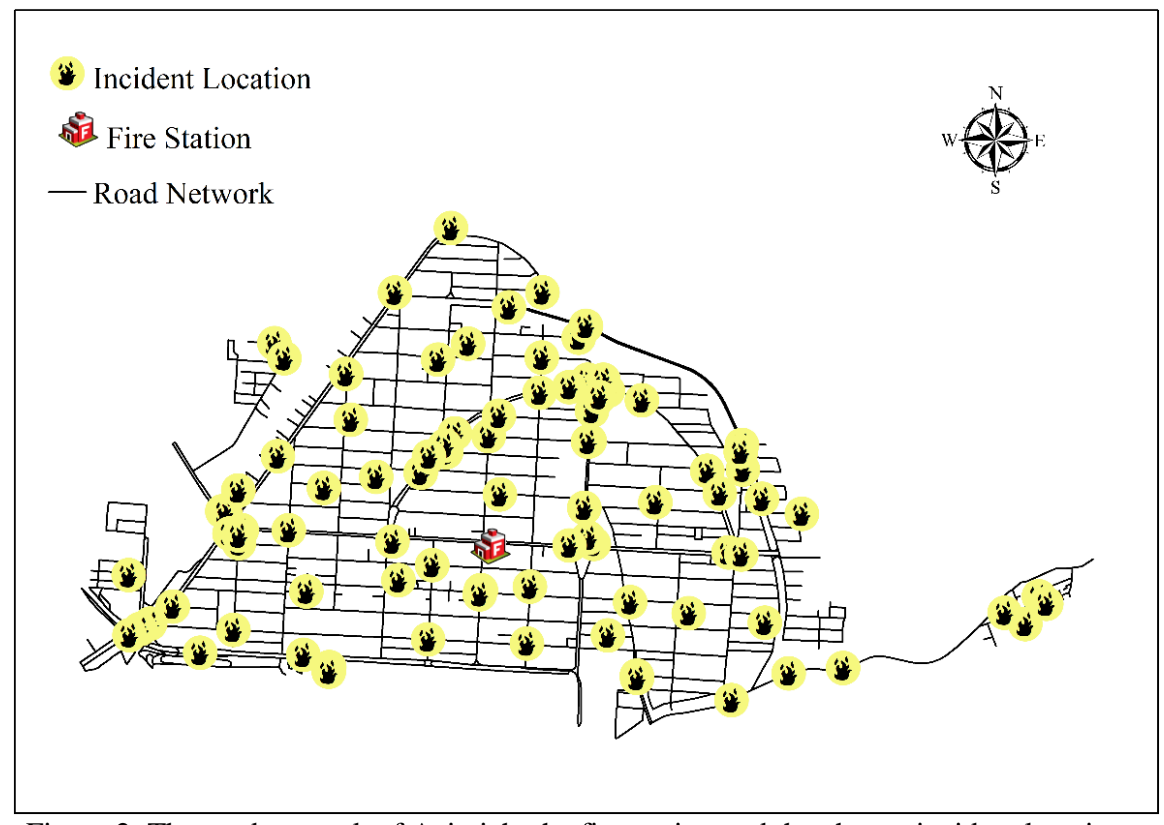

Figure 2. The road network of Azimieh, the fire station and the chosen incident locations.

\subsection{Shortest Path Results}

The proposed ant colony optimization is applied in accordance with the selected parameters in table 1. Performing ACO for an incident location, the pheromone values at each iteration change in such a way that the shortest paths are more likely to be selected. In other words, pheromone value on the constituent links of possible shorter paths is reinforced in the updating procedure while others loose importance due to evaporation. Figure 3 shows how pheromone values change as the algorithm advances.

\begin{tabular}{l|llll} 
Parameter & $\rho$ & $Q$ & $\alpha$ & Updating \\
\hline Value & 0.4 & $5 * \operatorname{Max}(\mathrm{L})$ & 5 & Iteration Best
\end{tabular}

Table1. Parameters defined for the experimented ACO solution.

The results of 15 selected items from all 88 paths are shown in table 2. The length of the paths from Dijkstra and the difference between Dijkstra and ACO is also stated.

In table 3, the sums of the length of all paths for both solutions are depicted. It implies that for a $164 \mathrm{~km}$ of all paths only $0.62 \mathrm{~km}$ extra length is imposed. It means that mistakes in finding the shortest path do not occur as an extremely long solution but a small detour from the best path. In other words, although ACO mistakes are not the shortest path but of the shortest on the top of the list. As shown in figure 4. The differences in the length of all 4 mistakes of ACO are not critical.

\begin{tabular}{|l|l|l|}
\hline Dijkstra(m) & ACO(m) & $\boldsymbol{\Delta d}(\mathbf{m})$ \\
\hline $\mathbf{2 0 8 4 . 2 2}$ & 2084.22 & 0 \\
\hline $\mathbf{1 9 9 2 . 3 8}$ & 1992.38 & 0 \\
\hline $\mathbf{1 9 5 1 . 1}$ & 1951.1 & 0 \\
\hline $\mathbf{1 0 9 . 4 8}$ & 109.48 & 0 \\
\hline $\mathbf{2 6 0 1 . 9}$ & 2601.9 & 0 \\
\hline $\mathbf{4 6 4 . 0 2}$ & 464.02 & 0 \\
\hline $\mathbf{1 0 7 1 . 8 1}$ & 1071.81 & 0 \\
\hline $\mathbf{1 4 8 1 . 4}$ & 1481.4 & 0 \\
\hline $\mathbf{1 4 0 4 . 2 1}$ & 1404.21 & 0 \\
\hline $\mathbf{1 8 5 5 . 5 8}$ & 1747.74 & 107.84 \\
\hline $\mathbf{1 3 2 8 . 7 5}$ & 1328.75 & 0 \\
\hline $\mathbf{7 6 1 . 7 2}$ & 761.72 & 0 \\
\hline $\mathbf{2 6 3 . 6 7}$ & 263.67 & 0 \\
\hline $\mathbf{3 9 4 . 4 6}$ & 394.46 & 0 \\
\hline $\mathbf{3 3 0 1 . 9 3}$ & 3301.93 & 0 \\
\hline
\end{tabular}

Table 2. The results of the shortest paths for 15 runs of the ACO and Dijkstra algorithms. 


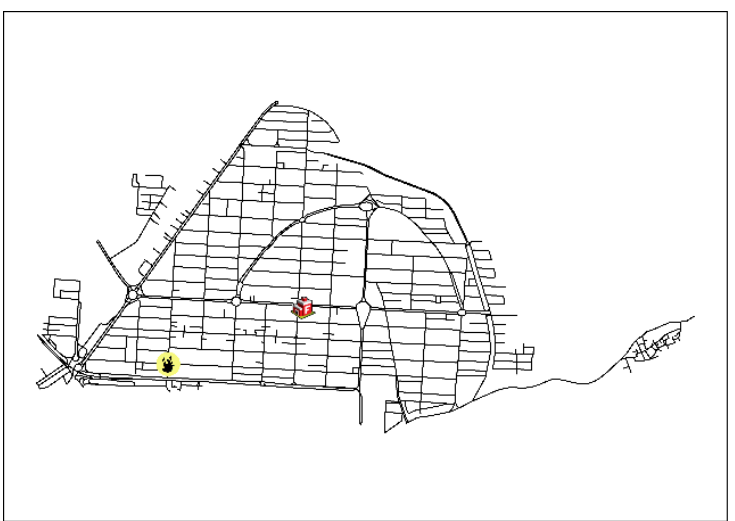

(a)

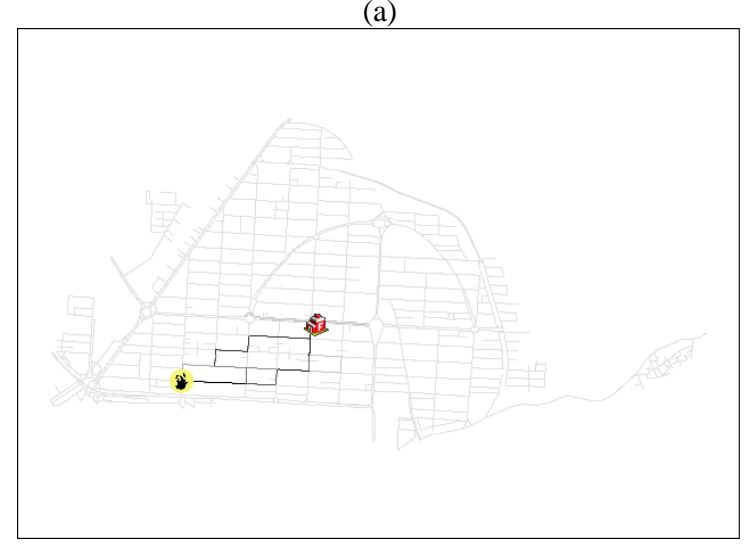

(c)

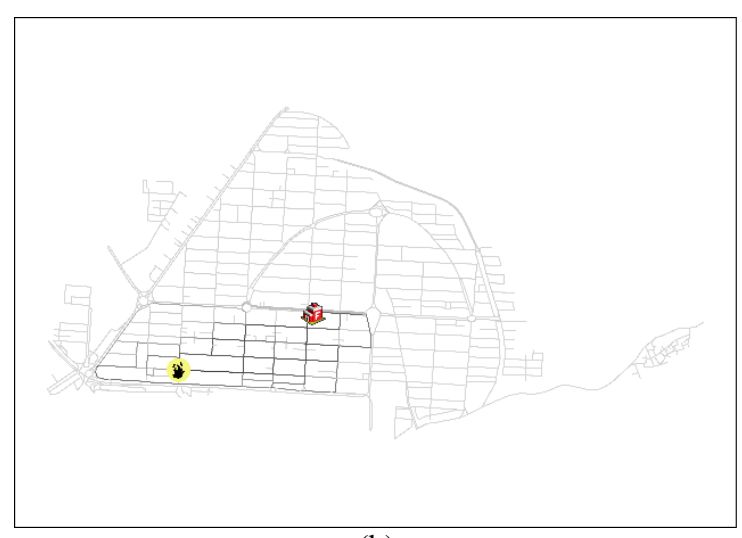

(b)

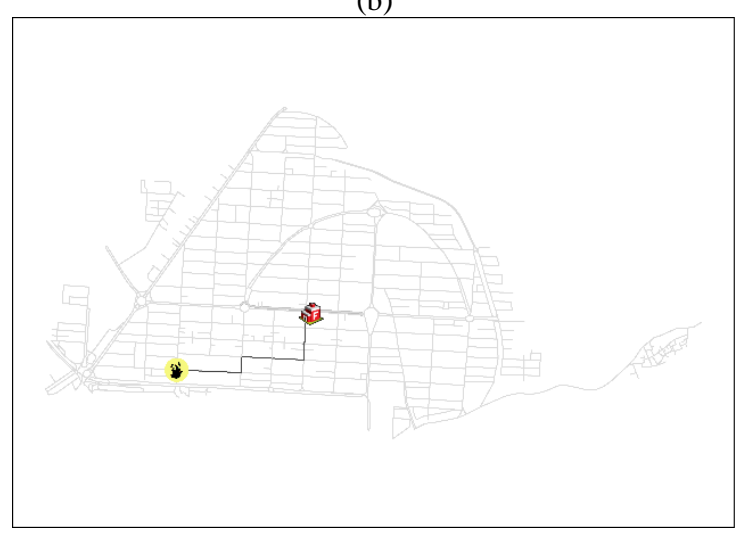

(d)

Figure 3. a) The pheromone value at the first iteration, b) The pheromone value at the second iteration, c) Pheromone value at 80 th iteration, d) The best path selected by ACO.

\begin{tabular}{c|lcc} 
& Dijkstra $(\mathrm{m})$ & $A C O(\mathrm{~m})$ & $\Delta d(\mathrm{~m})$ \\
\hline Length & 164078.62 & 164704.94 & 626.32 \\
Table3: & Sum of the length of the shortest path generated by
\end{tabular}
ACO and Dijkstra and the difference.

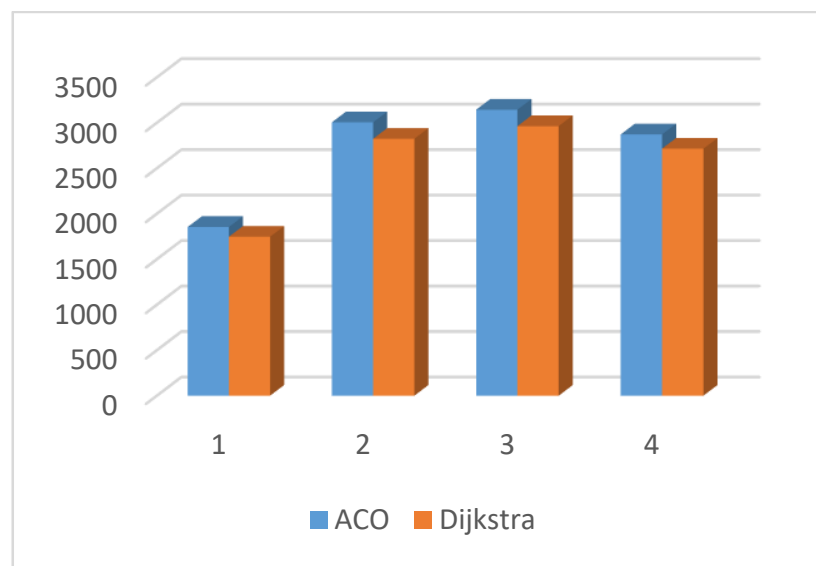

Figure 4 . The length of the 4 mistakes made by ACO compared to Dijkstra solution in meters.

\subsection{Critical Roads Results}

Finally, after computing all 88 paths, the pheromone values of the final iteration for each run of the algorithm are added together to generate the final sum of Pheromone. The results are shown in figure 5 .

Verification of the results seems to be an intricate situation. The first problem is the lack of reference ground truth of how critical roads are; as the whole concept of criticality is subjective concerning road network utilization purposes. In other words, the police station may classify a road segment as very critical, regarding their locations and service area but, the Fire station finds the criticality of the same road segment as Medium. Therefore, an inevitable solution for the verification of results is to refer to the firefighter of the station ask them to rate road segments in the region. The idea is recommended to be studied in future works.

For a situation like this visual inspection is always an option. Visual inspection shows that the results conform to the logic of accessibility as the main road where the fire station is located enjoys Very High pheromone value which means this road is very important for the execution of rescue missions. Moreover, the Chalous road which is the only option to reach Bilaqan region (an isolated region in the east) is also bolded relatively which confirms the validity of the results. Moreover, most main roads are highlighted as Medium and High which justifies the relevance of the results. 


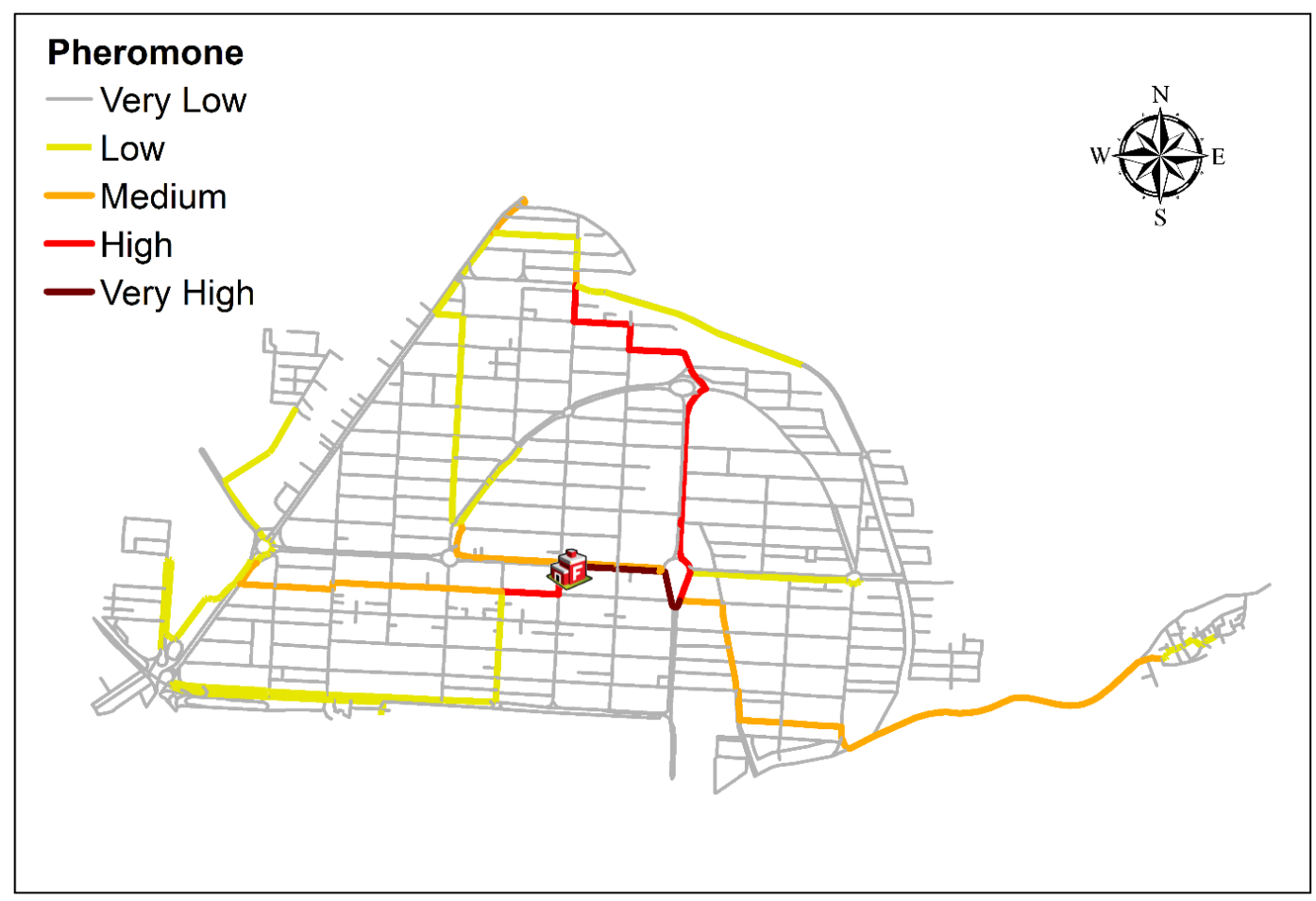

Figure 5. Categorizing road segments with respect to the pheromone values.

\subsection{Discussions}

Regrading to the capability of ACO to solve the shortest path problem, it is observed that the algorithm's success rate in finding the shortest path is $95.45 \%$. It is a general idea that conducting a comprehensive study to tune the ACO parameter would decrease the number of unsuccessful cases although in cases in which ACO fails to provide the shortest path, the extra meters are relatively small. This means that mistakes are some minor deviations from the best track, not huge leaps in the distance.

Considering elapsed time analysis, in case of small networks like the test study Dijkstra is more successful. Generally speaking, meta-heuristic algorithms such as ACO and genetic algorithms are not suitable for real-time problems. Therefore, the solution is recommended for the pre-event analysis of the network during the preparedness stage of the disaster management cycle.

Regarding the case study area, the resulted pheromone map reveals some critical issues about the fire stations' access to the region. Some secondary road segments are bolded which categorizes them as critical segments as they are important in terms of accessibility but should not be considered as a reliable choice due to their local access functionality as shown in figure 6a. Asbi square situation also seems critical as the results show high pheromone concentration around the square shown in Figure 6b. Although the existence of a U-turn at the entrance of the square looks promising, the traffic overflow at the square might jeopardize the safe and fast transportation of fire trucks. It is recommended to establish a U-turn prior to Asbi Square to avoid probable traffic congestion.

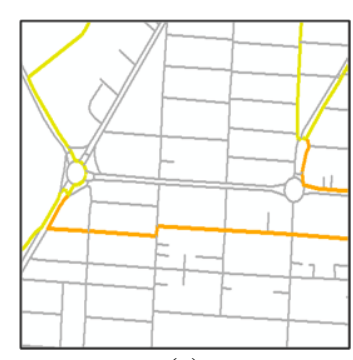

(a)

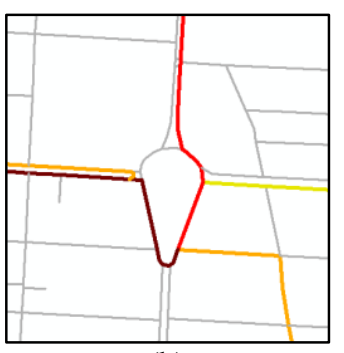

(b)
Figure 6.a) Some secondary roads with high pheromone concentration. b) Asbi square pheromone concentration.

\section{CONCLUSIONS}

This study considers the use of ACO for optimum path selection for rescue missions conducted by local fire stations. Therefore, the shortest paths (considering the length of the path as the cost) from the case study local fire station to the random incident locations are generated and compares to deterministic Dijkstra solution. Moreover, the sum of pheromone values as an indicator of the criticality of the road elements is discussed.

Regarding future works, it is recommended to consider travel time rather than the length of the path to solve the problem. It is also recommended to consider the traffic condition of the region to solve the problem. It is also recommended to study the possibility of generating a criticality map for different traffic zones such as morning and evening traffic congestion peaks.

\section{REFERENCES}


Behera, S., Bhattacharjee, S., Mishra, D., \& Panda, A., 2020. Application of Genetic Algorithm for Shortest Path Routing Problem. Sustainable Humanosphere, 16(1), 470-479 .

Bellman, R., 1958. On a routing problem. Quarterly of applied mathematics, 16: 87-90.

Cormen, T. H., Leiserson, C. E., Rivest, R. L., Stein, C., 2009. Introduction to algorithms: MIT press.

Dijkstra, E. W., 1959. A note on two problems in connexion with graphs. Numerische mathematik, 1(1), 269-271 .

Dorigo, M., 1992. Optimization, learning and natural algorithms [Ph. D. thesis]. Politecnico di Milano, Italy .

Dorigo, M., Di Caro, G., 1999. Ant colony optimization: a new meta-heuristic. Paper presented at the Proceedings of the 1999 congress on evolutionary computation-CEC99)-Cat. No. 99TH8406.

Engelbrecht, A. P., 2007. Computational intelligence: an introduction: John Wiley \& Sons.

Floyd, R. W., 1962. Algorithm 97: shortest path. Communications of the ACM, 5(6), 345 .

Forkuo, E. K., Quaye-Ballard, J. A., 2013. GIS based fire emergency response system .

Głabowski, M., Musznicki, B., Nowak, P., Zwierzykowski, P., 2012. Shortest path problem solving based on ant colony optimization metaheuristic. Image Processing \& Communications, 17(1-2), 7-17 .

Horoba, C., Sudholt, D., 2010. Ant colony optimization for stochastic shortest path problems. Paper presented at the Proceedings of the 12th annual conference on Genetic and evolutionary computation.

Johnson, D. B., 1977. Efficient algorithms for shortest paths in sparse networks. Journal of the ACM (JACM), 24(1), 1-13 .

Liu, Z., Kong, Y., Su, B., 2016. An improved genetic algorithm based on the shortest path problem. Paper presented at the 2016 IEEE international conference on information and automation (ICIA).

Sadhu, S., Singh, S. K., Kanga, S., 2017. Optimum path from tourist places to railway station and hospitals \& fire services using GIS-based network analysis: A case study of Jaisalmer and Sam Blocks of Jaisalmer District, Rajasthan (India). International Journal of Technology Research and Management, $4(8), 1-5$.

Samadzadegan, F., Zarrinpanjeh, N., Schenk, T., 2011. Ant colony optimization for finding best routes in disaster affected urban area. Paper presented at the 7th International Conference on Geoinformation for Disaster Management.

Sim, K. M., Sun, W. H., 2003. Ant colony optimization for routing and load-balancing: survey and new directions. IEEE transactions on systems, man, and cybernetics-Part A: systems and humans, 33(5), 560-572 .
Syarif, A., Muludi, K., Adrian, R., Gen, M., 2018. Solving fuzzy shortest path problem by genetic algorithm. Paper presented at the IOP Conference Series: Materials Science and Engineering.

Zeng, W., \& Church, R. L., 2009. Finding shortest paths on real road networks: the case for A. International journal of geographical information science, 23(4), 531-543 . 